\title{
Medium effect on the characteristics of the coupled seismic and electromagnetic signals
}

\author{
By Qinghua Huang, ${ }^{* 1, \dagger}$ Hengxin Ren, ${ }^{* 2}$ Dan ZHAnG $^{* 3}$ and Y. John CHEN*1 \\ (Communicated by Seiya UYEDA, M.J.A.)
}

\begin{abstract}
Recently developed numerical simulation technique can simulate the coupled seismic and electromagnetic signals for a double couple point source or a finite fault planar source. Besides the source effect, the simulation results showed that both medium structure and medium property could affect the coupled seismic and electromagnetic signals. The waveform of coupled signals for a layered structure is more complicated than that for a simple uniform structure. Different from the seismic signals, the electromagnetic signals are sensitive to the medium properties such as fluid salinity and fluid viscosity. Therefore, the co-seismic electromagnetic signals may be more informative than seismic signals.
\end{abstract}

Keywords: electrokinetic effect, medium effect, co-seismic electromagnetic signals, a double couple point model, a finite fault planar model

\section{Introduction}

Field experiments showed that the electric signals from shallow explosions could be detected by sensors on the ground surface. ${ }^{1)}$ This phenomenon is known as the seismo-electric effect. The reverse process, which is called the electro-seismic effect, was also confirmed by the field experiments of injecting electric current to the earth and measuring the ground vibration by geophones on the ground surface. ${ }^{1)}$ Known as a Onsager reciprocal relation, ${ }^{2)-4)}$ the above conversion effect of seismic and electromagnetic (EM) signals in porous media is useful for monitoring oil and gas reservoirs. ${ }^{1), 5), 6)}$

Besides the above conversion phenomena between seismic and EM signals, field observations also indicated the existence of the EM signals (or electric resistivity changes) accompanying with natural earthquakes (EQs). ${ }^{7)-13)}$ Such coupling phenomenon is usually called co-seismic effect, which has attracted common interest from both EM and seismological

\footnotetext{
*1 Department of Geophysics, School of Earth and Space Sciences, Peking University, Beijing 100871, China.

*2 School of Earth and Space Science, University of Science and Technology of China, Hefei 230026, China.

*3 Schlumberger, Beijing 100015, China.

$\dagger$ Correspondence should be addressed: Q. Huang, Department of Geophysics, School of Earth and Space Sciences, Peking University, Beijing 100871, China (e-mail: huangq@pku.edu.cn). Abbreviations: EQ: Earthquake; EM: electromagnetic.
}

communities. Because there are many reports on the EM signals preceding EQs, ${ }^{13)-19)}$ some scientists have tried to predict EQs based on pre-seismic EM signals. ${ }^{15), 20)-24)}$ Although there are some attempts of revealing the mechanisms of the pre-seismic EM signals on the basis of physical models, laboratory experiments and numerical simulations, ${ }^{15), 20), 25)-30 \text { ) }}$ the understanding of the seismic EM signals is not sufficient at the current stage. ${ }^{31)}$ Even the co-seismic effect may provide some useful information about the seismogenic process of EQs. ${ }^{29), 32)}$

As one of the possible generation mechanisms of the co-seismic EM signals, electrokinetic effect, 33 ),34) which satisfies the Onsager reciprocal relation, ${ }^{4)}$ has been paid much attention. The key concept underlying the electrokinetic effect is the electric double layer (Fig. 1). ${ }^{34)}$ It is well known that, in contact with a fluid electrolyte, most mineral grains develop a chemically and/or physically adsorbed surface charge which is electrically balanced by a diffuse layer of mobile counterions extending into the fluid phase. The adsorbed layer and diffuse layer together constitute the electric double layer (Fig. 1). Fluidpressure gradients and/or grain accelerations due to seismic waves can drive the flow of excess ions in diffuse layer. ${ }^{34), 35)}$

Combining the electrokinetic effect and the synthetic seismogram, Ren et al. ${ }^{36), 37)}$ developed an effective numerical technique for simulating the co- 


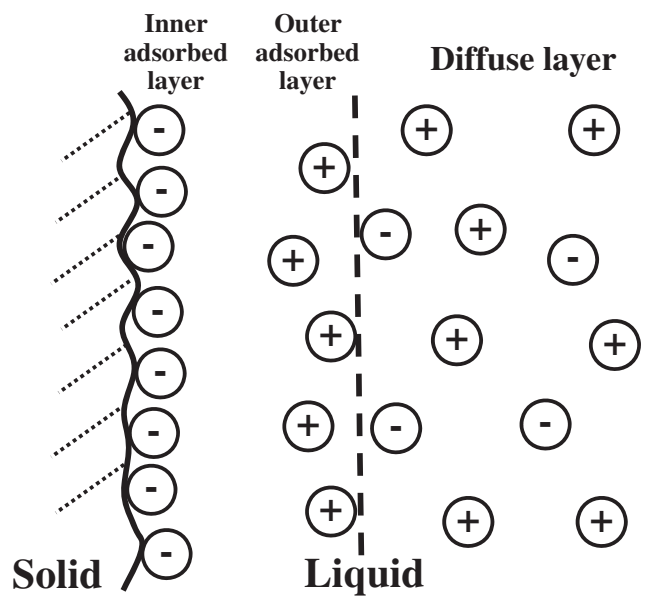

Fig. 1. A sketch of the electric double layer. The bold curve represents the solid-liquid surface. The vertical dashed line is the slipping plane.

seismic EM fields associated with seismic waves due to a point rupture of a double couple source or a planar rupture of a finite fault in layered porous media.

Using the above numerical simulation technique, Zhang et al. ${ }^{38)}$ investigated the coupled seismic and EM signals due to different source models, as well as different model structures. Generally, both seismic and EM signals were found to have simple waveforms for a simple source model, compared with that of the source model closer to a natural EQ.

In this study, continuing the work of Zhang et al., ${ }^{38)}$ we simulate the electrokinetically induced co-seismic EM signals for different medium models focusing on the effects of different properties of medium.

\section{Numerical simulation method of coupled seismic and EM signals}

The detailed information of the numerical simulation method is given in Ren et al. ${ }^{36), 37)}$ For readers' convenience, a brief description of the technique is summarized in Appendix A.

\section{Results}

Because this study focuses on the medium effects, for simplicity and easy comparison, all the simulations are based on a double couple point rupture source model.

Medium structure effect. In order to investigate the possible effect of medium structure on the coupled seismic and EM signals, we performed the simulations for different medium structures. As
Table 1. Properties of the porous media adopted in this study

\begin{tabular}{|c|c|c|c|c|}
\hline Properties & $\begin{array}{c}l_{1} \\
(\text { sand })\end{array}$ & $\begin{array}{c}l_{2} \\
(\text { sandstone })\end{array}$ & $l_{\text {strong }}$ & $l_{\text {weak }}$ \\
\hline Porosity $\phi, \%$ & 30 & 20 & 20 & 20 \\
\hline DC permeability $k_{0}, \mathrm{~m}^{2}$ & $10^{-11}$ & $10^{-12}$ & $10^{-12}$ & $10^{-12}$ \\
\hline Solid bulk modulus $k_{s}$, GPa & 36 & 36 & 36 & 80 \\
\hline Fluid bulk modulus $k_{f}, \mathrm{GPa}$ & 2.2 & 2.2 & 2.2 & 2.2 \\
\hline Frame bulk modulus $k_{f r}, \mathrm{GPa}$ & 0.4 & 5.0 & 5.0 & 5.0 \\
\hline Frame shear modulus $G, \mathrm{GPa}$ & 0.5 & 7.0 & 7.0 & 7.0 \\
\hline Fluid viscosity $\eta$, Pas & $10^{-3}$ & $10^{-3}$ & $10^{-5}$ & $10^{-3}$ \\
\hline Solid density $\rho_{s}, \mathrm{~kg} / \mathrm{m}^{3}$ & 2700 & 2700 & 2700 & 2700 \\
\hline Fluid density $\rho_{f}, \mathrm{~kg} / \mathrm{m}^{3}$ & 1000 & 1000 & 1000 & 1000 \\
\hline Salinity $C_{0}, \mathrm{~mol} / \mathrm{L}$ & $10^{-3}$ & $10^{-3}$ & $10^{-6}$ & $10^{-3}$ \\
\hline Temperature $T, \mathrm{~K}$ & 298 & 298 & 298 & 298 \\
\hline Fluid Permittivity $\kappa_{f}$ & 80 & 80 & 80 & 80 \\
\hline Solid Permittivity $\kappa_{s}$ & 4 & 4 & 4 & 4 \\
\hline Tortuosity $\alpha_{\infty}$ & 3 & 3 & 3 & 3 \\
\hline
\end{tabular}

an example, we chose a full space, a half space, and a two-layer model with a double couple point rupture source (Fig. 2). The double couple point source applied here is $\boldsymbol{M}_{x z}+\boldsymbol{M}_{z x}$, which can represent either a fault in $x-0-y$ plane with slip displacement in $x$ direction or a fault in $y-o-z$ plane with slip displacement in $z$-direction. The amplitudes of the two seismic moments are $M_{x z}=M_{z x}=1.12 \times 10^{14} \mathrm{~N} \cdot \mathrm{m}$. The source is at the position of $(0,0,5) \mathrm{km}$, and the receiver is set at $(1,0.5,0.05) \mathrm{km}$. The medium parameters are given in Table 1 . We chose sandstone $\left(l_{2}\right)$ and sand $\left(l_{1}\right)$ to construct the medium structure models shown in Fig. 2a, 2b and 2c.

Figure 3 shows the calculated $x$-component of seismic field from the different structure models given in Fig. 2. Marks a, b and c are common to both Fig. 2 and Fig. 3. One can see much complicated waveforms for the layered model (Fig. 3c) due to the multiple reflections of seismic waves.

Figure 4 gives the waveforms of the $x$-component of electric field calculated from the same full space, half space, and two-layer models shown in Fig. 2a, 2b and 2c. In these figures, rectangles show the rupture signals as explained later. In the full space model, the electric field accompanying with the seismic P-wave is stronger than that of the S-wave (Fig. 4a). This result is consistent with the general knowledge that the seismic $\mathrm{P}$-wave can induce electric signals much stronger than that of the Swave. ${ }^{35)-37), 39)}$

However, in most of the field observations for natural earthquakes, electric signals due to the S- 


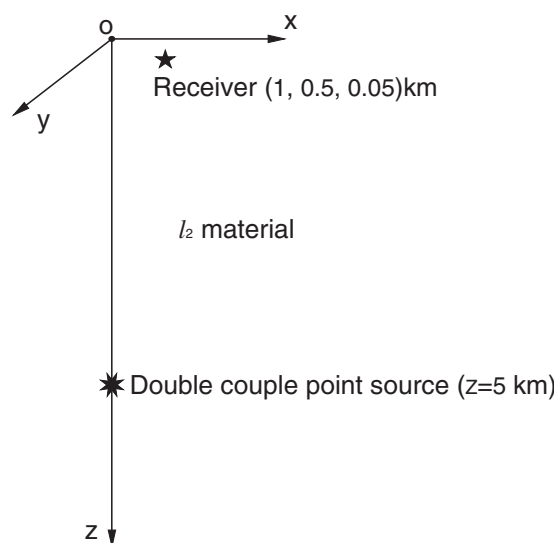

(a)

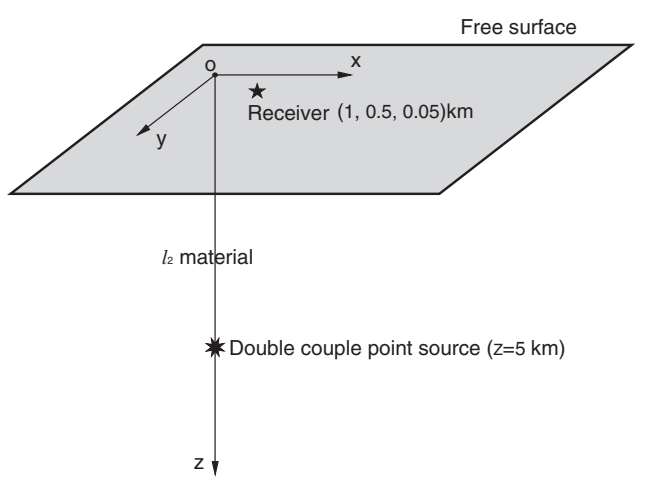

(b)

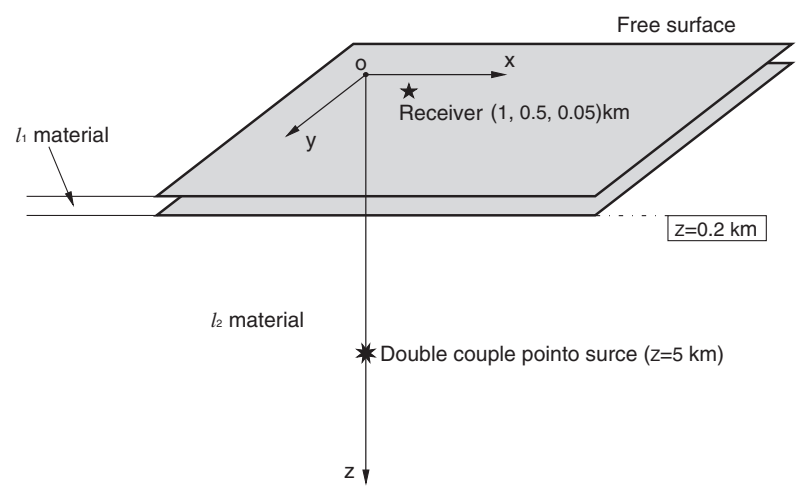

(c)

Fig. 2. The model configuration in this study. (a) A full space, (b) a half space, and (c) a two-layer model with a double couple point rupture source. The medium parameters of $l_{1}$ (sand) and $l_{2}$ (sandstone) are given in Table 1.

wave have been more clearly seen than those due to the P-wave. ${ }^{7), 8), 10), 12), 13) ~ S i m i l a r ~ r e s p o n s e ~ h a s ~ b e e n ~}$ also confirmed by our simulation results for a half space model (Fig. 4b) and a two-layer model (Fig. 4c). It should be mentioned that the field observations, Fig. 4b and Fig. 4c do not mean the
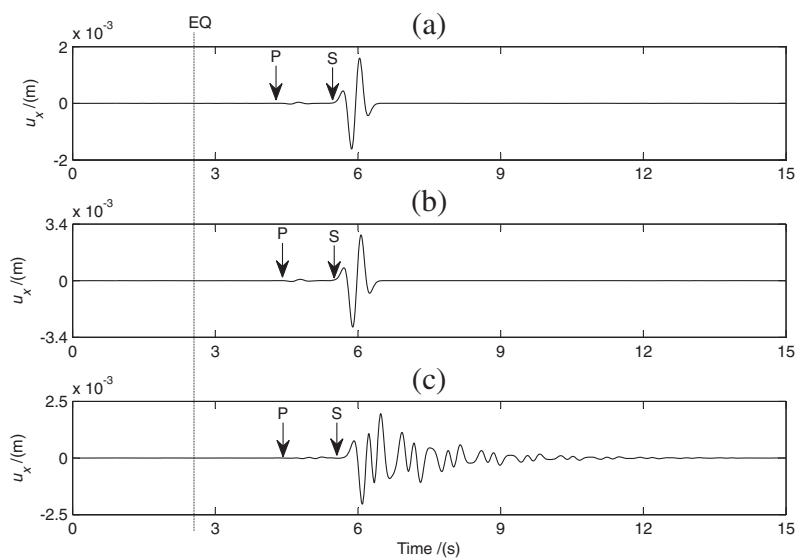

Fig. 3. The waveform of $u_{x}$ (the horizontal $x$-component of seismic field) of (a) a full space, (b) a half space, and (c) a twolayer model with a double couple point rupture source. The model configuration is given in Fig. 2. The medium parameters are given in Table 1. The vertical dash line with "EQ" shows the rupture time. The arrows with "P" and "S" indicate the arrival times of $\mathrm{P}$-wave and $\mathrm{S}$-wave.
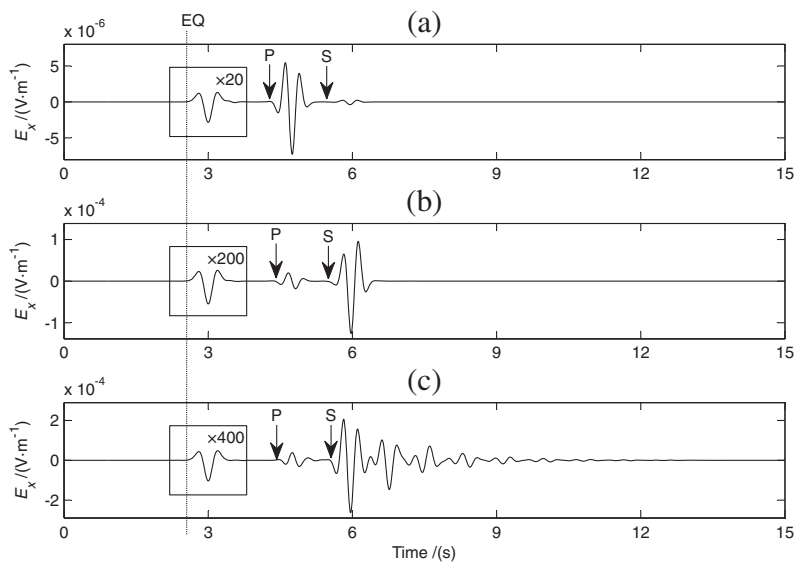

Fig. 4. The waveform of $E_{x}$ (the horizontal $x$-component of electric field) of (a) a full space, (b) a half space, and (c) a twolayer model with a double couple point rupture source. The model configuration is given in Fig. 2. The medium parameters are given in Table 1 . The weak electric signals directly induced by the double couple source at the rupture time are amplified in the rectangles to enhance the visibility. The numbers in the rectangles mean the related amplification factor. The vertical dash line with "EQ" shows the rupture time. The arrows with "P" and "S" indicate the arrival times of P-wave and S-wave.

S-wave has a higher capability in inducing electric signals than the $\mathrm{P}$-wave, i.e., the phenomena are not contradicting with Fig. 4a and the related general knowledge. ${ }^{35)-37), 39)}$ In fact, the difference in Fig. 4a and Fig. 4b/Fig. 4c is due to the existence of Sv-to-P wave converted on the surface of the half space, i.e., 
it is the $\mathrm{Sv}$-to-P converted wave rather than the Swave itself which induces the strong electric signals at the arrival time of the $\mathrm{S}$-wave. This is the reason why in most of the field observations for natural earthquakes similar responses have been clearly seen, because in the nature there is a surface on the ground and the seismic Sv-wave can be converted to the Pwave on the ground surface.

Compared with the simple full space and half space models, the layered model leads to a more complicated waveform of the induced electric signals even for a simple point source model (Fig. 4c). The complicated waveform in the layered model is due to the multiple reflections in the layered model.

It should be mentioned that besides the EM signals accompanying with the seismic waves, some weak co-rupture EM signals prior to the arrival of seismic waves are induced directly by the double couple point source. Figure 4 shows the $x$-component of the co-rupture electric signals, which are amplified in the rectangles in Fig. 4 . The numbers in the rectangles mean the related amplification factor.

Medium property effect. We performed the simulations for different models to investigate the possible medium property effect. For simplicity, we adopted a double couple point source in a full space as examples. We set the double couple point source at the position of $(0,0,10) \mathrm{km}$, and the receiver at $(3,4,0.05) \mathrm{km}$. The other model configuration is the same as in Fig. 2a. The medium is sandstone whose properties are given in Table 1 (see the column marked by $l_{2}$ ).

For different values of fluid salinity (keep other parameters unchanged, i.e., the same as the reference parameters of sandstone $\left(l_{2}\right)$ in Table 1$)$, one can obtain the waveforms of each component of the seismic vibration velocity, acceleration, electric field, and magnetic field. Figure 5 shows the results of the $x$-component. The vibration velocity and acceleration keep unchanged for different values of salinity (Fig. 5a, b). There are clear electric signals accompanying with the seismic P-wave (Fig. 5c) and magnetic signals accompanying with the seismic Swave (Fig. 5d), consistent with the numerical results obtained in literatures. ${ }^{35)-37)}$ The phases of the EM signals are not sensible to the variation of salinity. However, the amplitudes of both the electric and magnetic signals depend significantly on the fluid salinity. The smaller the salinity is, the bigger the amplitude will be.

In order to quantify the salinity effect, we investigate the maximum amplitudes due to different

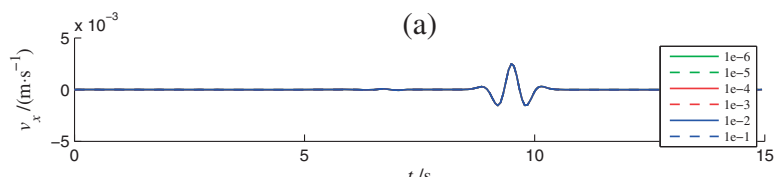

(b)
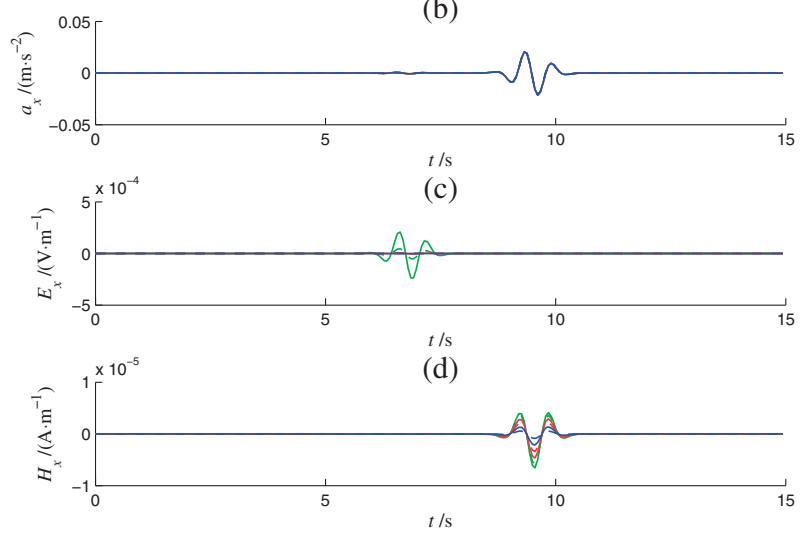

Fig. 5. The salinity-dependent waveform of the $x$-component of (a) vibration velocity, (b) vibration acceleration, (c) electric field, and (d) magnetic field.

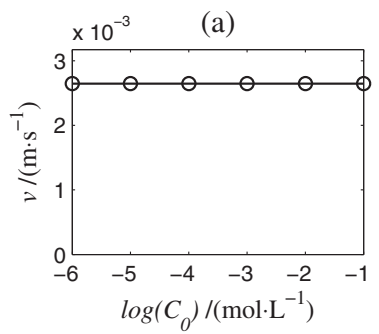

(b)
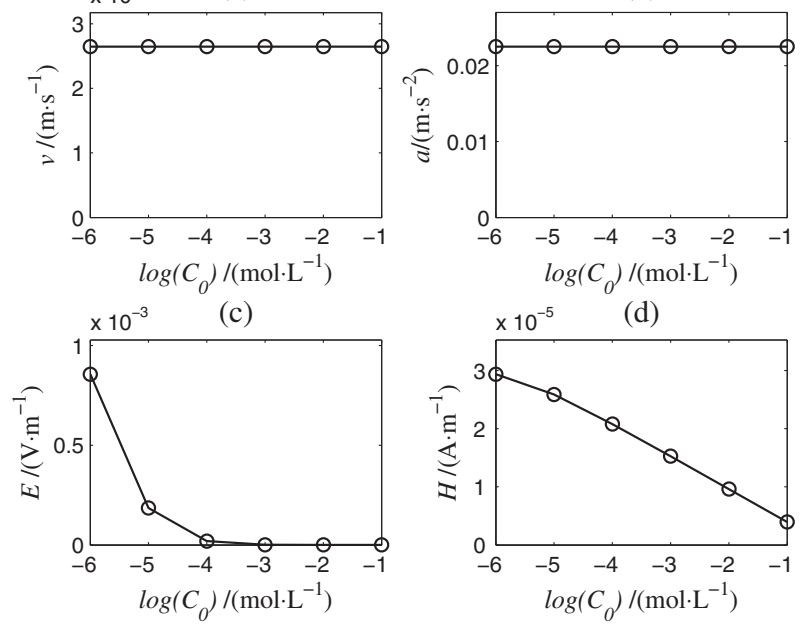

Fig. 6. The salinity-dependent amplitudes of (a) vibration velocity, (b) vibration acceleration, (c) electric field, and (d) magnetic field.

values of salinity. The results are summarized in Fig. 6. It is confirmed that the maximum amplitudes of both the velocity and the acceleration are not affected by salinity (Fig. 6a, b). On the other hand, the amplitudes of both the electric and magnetic fields decrease with increase of salinity (Fig. 6c, d).

We repeated our simulations for the models with different values of fluid viscosity. We show the results in Fig. 7. Similarly to the salinity effect, neither the velocity nor the acceleration is sensible to the 

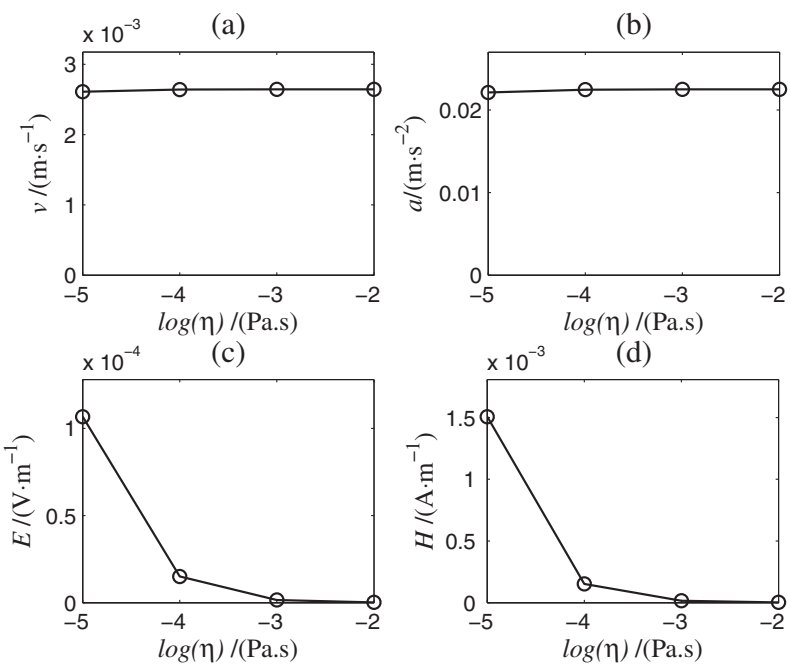

Fig. 7. The viscosity-dependent amplitudes of (a) vibration velocity, (b) vibration acceleration, (c) electric field, and (d) magnetic field.

variation of viscosity (Fig. 7a, b). No viscositydependence is found for the phases of the EM field. However, the amplitudes of both the electric and magnetic fields decrease significantly with increase of viscosity (Fig. 7c, d).

\section{Discussion and conclusion}

Zhang et $a l .{ }^{38)}$ investigated the coupled seismic and EM signals emitted from different source models. They found that both seismic and EM signals have simple waveforms for simple source models (e.g., a point rupture source, or a source time function of Ricker wavelet). More complicated waveforms are obtained for the source models closer to those of natural EQs, e.g., a planar rupture source, or a trapezoid or Herrmann source time function.

We simulated the characteristics of the coupled seismic and EM signals, based on our numerical method. ${ }^{36), 37)}$ The simulation results of different models indicated that the co-seismic EM signals depend on the model structure. Complex structure leads to more complicated waveform of the induced EM signals, possibly due to the multiple reflection effects (Fig. 4). Comparing with the current results in Fig. 4 and those of Zhang et al. ${ }^{38)}$ we found that stronger EM signals can be expected for the model with either a shallower seismic source or a smaller source-receiver distance.

Our results showed that medium effects may amplify the co-seismic EM signals, leading to the possible selectivity phenomenon. Selectivity phe- nomenon was first found for seismic electric signal (SES), which is a hot topic receiving active debates. $^{20), 21), 25)-28), 31)}$ This study may provide some information for the possible selectivity of co-seismic EM signals. Intensive investigation of the multicontributions from different medium parameters with a complicated medium model deserves further efforts. As an example, we calculated two cases with different values of fluid salinity, viscosity and solid bulk modulus in a full space model and a half space model, respectively. The double couple point source applied here is $\boldsymbol{M}_{x z}+\boldsymbol{M}_{z x}$, with $M_{x z}=M_{z x}=1.12 \times$ $10^{14} \mathrm{~N} \cdot \mathrm{m}$. The source is at the position of $(0,0,5) \mathrm{km}$, and the receiver is set at $(1,0.5,0.05) \mathrm{km}$. The medium parameters are listed in Table 1 (see columns $l_{\text {strong }}$ and $l_{\text {weak }}$ ). Figure 8 and Fig. 9 show the seismic, electric and magnetic wavefields of the full space model. The results indicated that the EM wavefields with the medium parameters of $l_{\text {strong }}$ in Table 1 (Fig. 8) could be 2-4 orders stronger than those with the medium parameters of $l_{\text {weak }}$ in Table 1 (Fig. 9), even the seismic wavefields are at the same order for both medium parameters of $l_{\text {strong }}$ and $l_{\text {weak }}$. Such conclusion also holds for the half space model. These results may provide some insights of the future field observations, such as the difference in the detectability of co-seismic EM signals in different tectonic background and medium structures.

It should be mentioned that besides the EM signals accompanying with the seismic waves, some EM signals prior to the arrival of seismic waves are induced directly by the double couple point model (Fig. 4). In order to distinguish the above two kinds of EM signals, we called the former as co-seismicwave EM signals and the latter as co-rupture EM signals. As indicated by our model results (Fig. 4), the co-rupture EM signals are generally much weaker than the co-seismic-wave EM signals. This might be the reason why there are numerous reports on coseismic-wave EM signals, while no reports on corupture EM signals of natural earthquakes so far.

In summary, our numerical simulations indicated that neither seismic vibration velocity nor acceleration is sensible to the variations of salinity or viscosity. However, the amplitudes of the EM signals decrease with the increase of both salinity and viscosity. The medium effects indicated that the coseismic EM signals could be amplified 2-4 orders, even the related seismic wavefields are at the same order. Such results may provide some insights of understanding the difference in the detectability of co-seismic EM signals in different geological regions. 

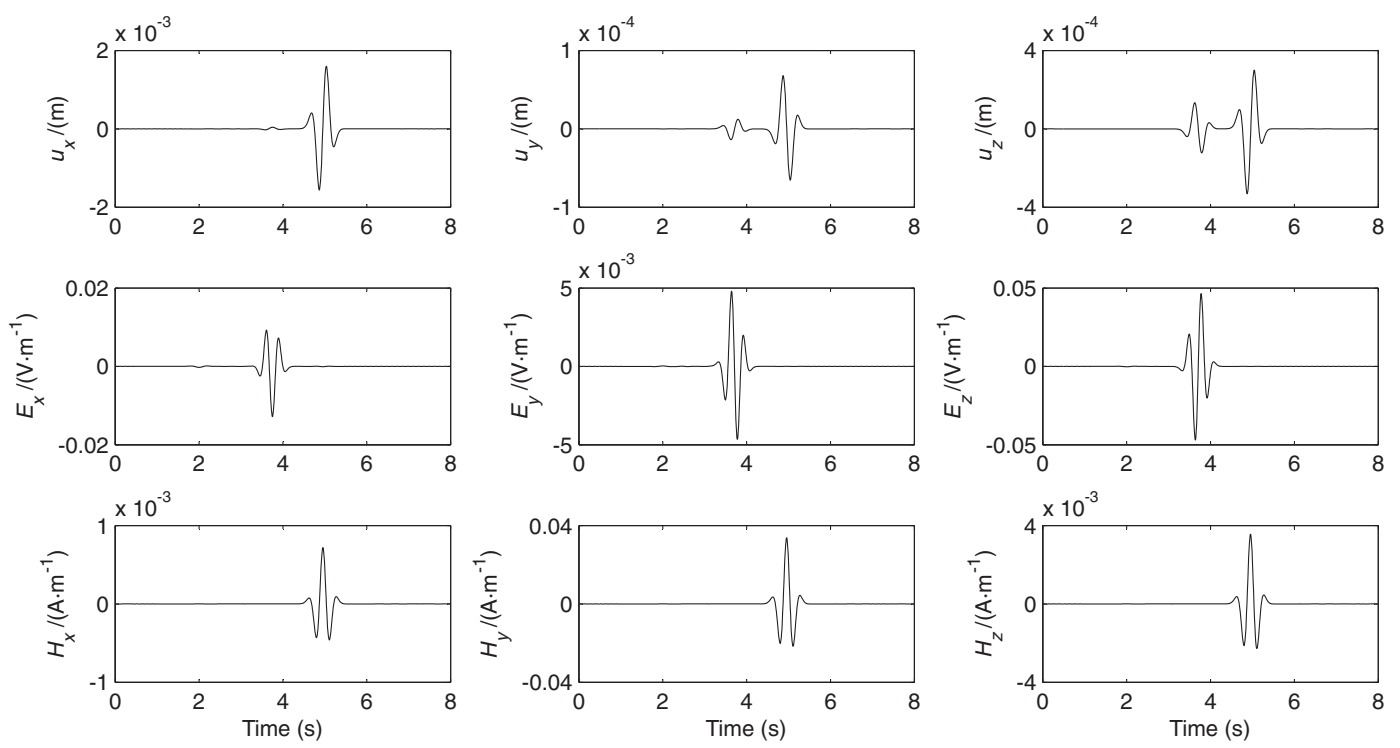

Fig. 8. The seismic, electric and magnetic wavefields of a full space model (the model configuration is given in Fig. 2a) with the medium parameters of $l_{\text {strong }}$ in Table 1.
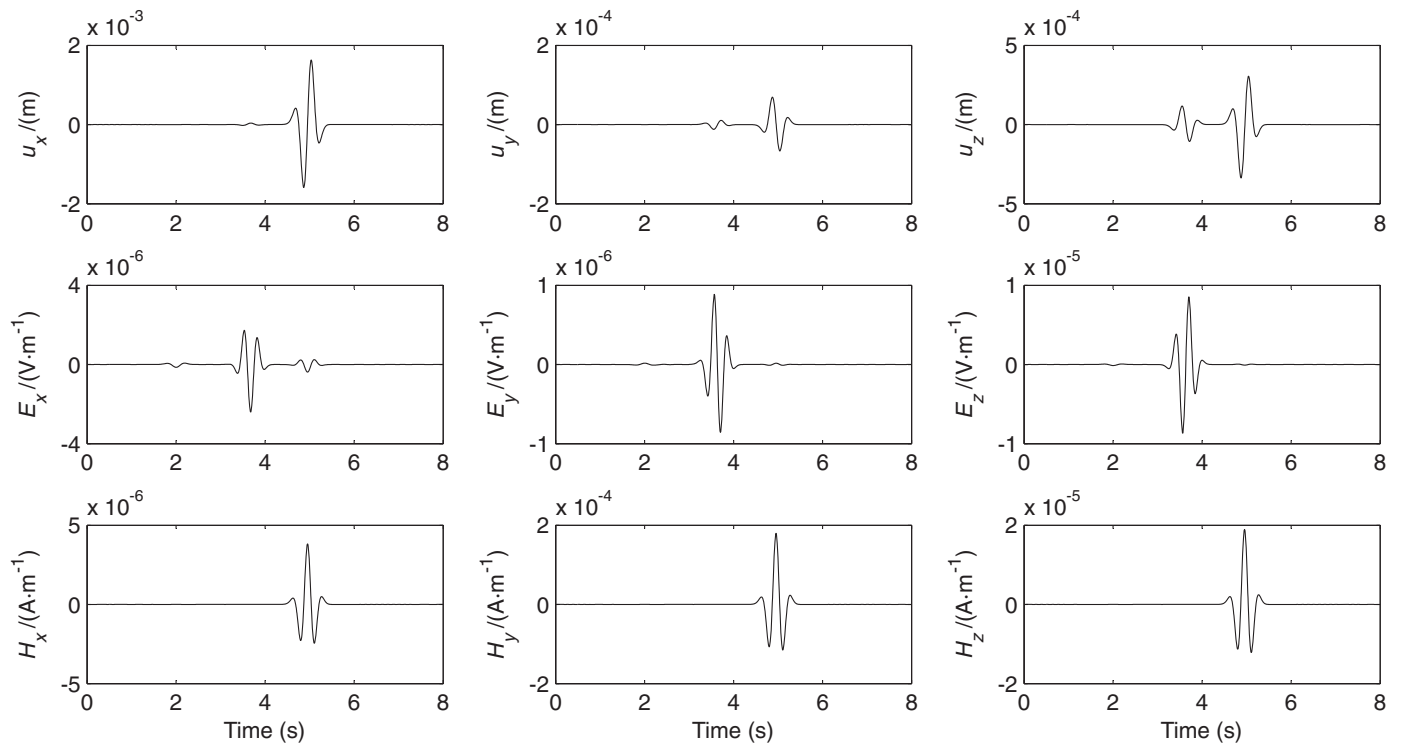

Fig. 9. The seismic, electric and magnetic wavefields of a full space model (the model configuration is given in Fig. 2a) with the medium parameters of $l_{\text {weak }}$ in Table 1 .

\section{Appendix A}

For a layered porous media with a source in the $s$-th layer, the scalar and vector functions involved in the governing equations of seismic and EM wavefields are expanded in a cylindrical coordinate system. ${ }^{36}$ Two sets of linear ordinary differential equations were obtained, corresponding to the case of SHTE ( $\mathrm{SH}$ waves generating electrical currents in the $\mathrm{SH}$ particle motion plane and the currents couple to the EM-wavefield components with transverse electric polarization) and the case of PSVTM (P and Sv waves generating electric currents in the PSV particle motion plane and the currents couple to the EMwavefield components with transverse magnetic polarization). Both cases have the following form in the $j$-th layer, ${ }^{37)}$ 


$$
\begin{aligned}
\frac{\partial}{\partial z} \mathbf{y}^{(j)}(z) & =\mathbf{A}^{(j)} \mathbf{y}^{(j)}(z)+\delta_{j, s} \mathbf{F}(z), \\
\text { for } z^{(j-1)} & <z<z^{(j)}
\end{aligned}
$$

where $\mathbf{y}$ is the vector of the corresponding expansion coefficients of unknown vector functions, $\mathbf{F}$ is the vector of the source information, $\mathbf{A}$ is a matrix, and $\delta_{j . s}$ is Kronecker delta function. The explicit expressions of $\mathbf{y}, \mathbf{F}$, and $\mathbf{A}$ are given in Ren et al. ${ }^{37)}$

Solving the above equations by using the generalized reflection and transmission coefficients method, ${ }^{40)}$ one can obtain the general solution in the following form, ${ }^{37)}$

$$
\mathbf{y}^{(j)}(z)=\Theta^{(j)} \Lambda^{(j)}(z)\left[\mathbf{a}^{(j)}+\delta_{j, s} \mathbf{b}(z)\right]
$$

where the wave-amplitude vector $\mathbf{a}$ is an unknown constant vector to be determined, $\mathbf{b}$ is the source term vector, $\boldsymbol{\Theta}$ and $\boldsymbol{\Lambda}$ are the eigen-decompositions of matrix A. Their detailed expressions are given in Ren et $a l .{ }^{37)}$

After obtaining the wave-field solutions in the frequency-wavenumber domain, we can transform them back to the space-time domain by using the well-known discrete wavenumber method, ${ }^{40)}$ which uses a spatial periodicity of sources to discretize the radiated wave-fields and imposes the discrete Fourier transform with complex frequencies to carry out the integration over frequency domain.

\section{Acknowledgements}

This study is supported by the National Science Fund for Distinguished Young Scholars (41025014), the China-Korea-Japan Joint Research Collaboration Program by the Ministry of Science and Technology of China (2010DFA21570), and the Research Fund for the Doctoral Program of Higher Education, Ministry of Education, China (20120001110073). The authors thank Prof. Seiya Uyeda, M.J.A., and the anonymous referees for the insightful comments and discussions.

\section{References}

1) Thompson, A.H. and Gist, G.A. (1993) Geophysical applications of electrokinetic conversion. Leading Edge 12, 1169-1173.

2) Onsager, L. (1931) Reciprocal relations in irreversible processes. I. Phys. Rev. 37, 405-426.

3) Onsager, L. (1931) Reciprocal relations in irreversible processes. II. Phys. Rev. 38, 2265-2279.

4) Miller, D.G. (1960) Thermodynamics of irreversible processes. The experimental verification of the Onsager reciprocal relations. Chem. Rev. 60, 1537 .
5) Thompson, A.H., Hornbostel, S., Burns, J., Murray, T., Raschke, R., Wridel, J., McCammon, P., Sumner, J., Haake, G. and Bixby, M. (2007) Field tests of electroseismic hydrocarbon detection. Geophysics 72, N1-N9.

6) Haines, S.S., Pride, S.R., Klemperer, S.L. and Biondi, B. (2007) Seismoelectric imaging of shallow targets. Geophysics 72, G9-G20.

7) Nagao, T., Orihara, Y., Yamaguchi, T., Takahashi, I., Hattori, K., Noda, Y. and Sayanagi, K. (2000) Co-seismic geoelectric potential changes observed in Japan. Geophys. Res. Lett. 27, 1535-1538.

8) Skordas, E., Kapiris, P., Bogris, N. and Varotsos, P. (2000) Field experimentation on the detectability of co-seismic electric signals. Proc. Jpn. Acad., Ser. B 76, 51-56.

9) Matsushima, M., Honkura, Y., Oshiman, N., Baris, S., Tuncer, M.K., Tank, S.B., Celik, C., Takahashi, F., Nakanishi, M., Yoshimura, R., Pektas, R., Komut, T., Tolak, E., Ito, A., Iio, Y. and Isikara, M. (2002) Seismoelectromagnetic effect associated with the Izmit earthquake and its aftershocks. Bull. Seismol. Soc. Am. 92, 350-360, doi:10.1785/ 0120000807.

10) Honkura, Y., Ogawa, Y., Matsushima, M., Nagaoka, S., Ujihara, N. and Yamawaki, T. (2009) A model for observed circular polarized electric fields coincident with the passage of large seismic waves. J. Geophys. Res. 114, B10103, doi:10.1029/ 2008JB006117.

11) Honkura, Y., Oshiman, N., Matsushima, M., Barıs, S., Tuncer, M.K., Tank, S.B., Celik, C. and Ciftci, E.T. (2013) Rapid changes in the electrical state of the 1999 Izmit earthquake rupture zone. Nat. Commun. 4, 2116, doi:10.1038/ncomms3116.

12) Tang, J., Zhan, Y., Wang, L.F., Dong, Z.Y., Zhao, G.Z. and Xu, J.L. (2010) Electromagnetic coseismic effect associated with aftershock of Wenchuan Ms 8.0 earthquake. Chin. J. Geophys. 53, 526-534.

13) Huang, Q.H. (2011) Retrospective investigation of geophysical data possibly associated with the Ms8.0 Wenchuan earthquake in Sichuan China. J. Asian Earth Sci. 41, 421-427.

14) Nagao, T., Enomoto, Y., Fujinawa, Y., Hata, M., Hayakawa, M., Huang, Q., Izutsu, J., Kushida, Y., Maeda, K., Oike, K., Uyeda, S. and Yoshino, T. (2002) Electromagnetic anomalies associated with 1995 Kobe earthquake. J. Geodyn. 33, 401-411.

15) Uyeda, S., Nagao, T. and Kamogawa, M. (2009) Short-term earthquake prediction: current status of seismo-electromagnetics. Tectonophysics 470, 205-213.

16) Han, P., Huang, Q.H. and Xiu, J.G. (2009) Principal component analysis of geomagnetic diurnal variation associated with earthquakes: case study of the M6.1 Iwate-ken Nairiku Hokubu earthquake. Chin. J. Geophys. 52, 1556-1563.

17) Han, P., Hattori, K., Huang, Q.H., Hirano, T., Ishiguro, Y., Yoshino, C. and Febriani, F. (2011) Evaluation of ULF electromagnetic phenomena associated with the 2000 Izu Islands earthquake swarm by wavelet transform analysis. Nat. 
Hazards Earth Syst. Sci. 11, 965-970.

18) Xu, G.J., Han, P., Huang, Q.H., Hattori, K., Febriani, F. and Yamaguchi, H. (2013) Anomalous behaviors of geomagnetic diurnal variations prior to the 2011 off the Pacific coast of Tohoku earthquake (Mw9.0). J. Asian Earth Sci. 77, 5965 .

19) Hattori, K., Han, P., Yoshino, C., Febriani, F., Yamaguchi, H. and Chen, C.H. (2013) Investigation of ULF seismo-magnetic phenomena in Kanto, Japan during 2000-2010: case studies and statistical studies. Surv. Geophys. 34, 293-316.

20) Varotsos, P. (2005) The Physics of Seismic Electric Signals. Terrapub, Tokyo.

21) Varotsos, P. and Lazaridou, M. (1991) Latest aspects of earthquake prediction in Greece based on seismic electric signals. Tectonophysics 188, 321347.

22) Uyeda, S., Nagao, T., Orihara, Y., Yamaguchi, T. and Takahashi, I. (2000) Geoelectric potential changes: possible precursors to earthquakes in Japan. Proc. Natl. Acad. Sci. U.S.A. 97, 45614566.

23) An, Z.H., Du, X.B., Tan, D.C., Fan, Y.Y., Liu, J. and Cui, T.F. (2013) Study on the geo-electric field variation of Sichuan Lushan Ms7.0 and Wenchuan Ms8.0 earthquake. Chin. J. Geophys. 56, 38683876 .

24) Sobolev, G.A. (1975) Application of electric method in the tentative short-term forecast of Kamchatka earthquake. Pure Appl. Geophys. 113, 229-235.

25) Huang, Q. and Ikeya, M. (1998) Seismic electromagnetic signals (SEMS) explained by a simulation experiment using electromagnetic waves. Phys. Earth Planet. Inter. 109, 107-114.

26) Huang, Q. and Ikeya, M. (1999) Experimental study on the propagation of seismic electromagnetic signals (SEMS) using a mini-geographic model of the Taiwan strait. Episodes 22, 289-294.

27) Huang, Q.H. and Lin, Y.F. (2010) Selectivity of seismic electric signal (SES) of the $2000 \mathrm{Izu}$ earthquake swarm: a 3D FEM numerical simulation model. Proc. Jpn. Acad., Ser. B 86, 257-264.

28) Huang, Q.H. and Lin, Y.F. (2010) Numerical simulation of selectivity of seismic electric signal and its possible influences. Chin. J. Geophys. 53,
$535-543$.

29) Huang, Q.H. (2011) Rethinking earthquake-related DC-ULF electromagnetic phenomena: towards a physics-based approach. Nat. Hazards Earth Syst. Sci. 11, 2941-2949.

30) Freund, F. (2010) Toward a unified solid state theory for pre-earthquake signals. Acta Geophys. 58, 719-766.

31) Lighthill, S.J. (1996) A Critical Review of VAN: Earthquake Prediction from Seismic Electrical Signals. World Scientific Press, Singapore.

32) Huang, Q.H. (2002) One possible generation mechanism of co-seismic electric signals. Proc. Jpn. Acad., Ser. B 78, 173-178.

33) Mizutani, H., Ishido, T., Yokokura, T. and Ohnishi, S. (1976) Electrokinetic phenomena associated with earthquake. Geophys. Res. Lett. 3, 365-368.

34) Pride, S.R. (1994) Governing equations for the coupled electromagnetics and acoustics of porous media. Phys. Rev. B 50, 15678-15696.

35) Garambois, S. and Dietrich, M. (2001) Seismoelectric wave conversions in porous media: field measurements and transfer function analysis. Geophysics 66, 1417-1430.

36) Ren, H.X., Huang, Q.H. and Chen, X.F. (2010) A new numerical technique for simulating the coupled seismic and electromagnetic waves in layered porous media. Earthq. Sci. 23, 167-176.

37) Ren, H.X., Chen, X.F. and Huang, Q.H. (2012) Numerical simulation of co-seismic electromagnetic fields associated with seismic waves due to finite faulting in porous media. Geophys. J. Int. 188, 925-944.

38) Zhang, D., Ren, H.X. and Huang, Q.H. (2013) Numerical simulation study of co-seismic electromagnetic signals in porous media. Chin. J. Geophys. 56, 2739-2747.

39) Haartsen, M.W. and Pride, S.R. (1997) Electroseismic waves from point sources in layered media. J. Geophys. Res. 102, 24745-24769.

40) Bouchon, M. (2003) A review of the discrete wavenumber method. Pure Appl. Geophys. 160, 445466 .

(Received July 14, 2014; accepted Nov. 25, 2014) 